\title{
Notch3 signaling-mediated melanoma-endothelial crosstalk regulates melanoma stem-like cell homeostasis and niche morphogenesis
}

\author{
Mei-Yu Hsu, Moon Hee Yang, Caroline I Schnegg, Soonyean Hwang, Byungwoo Ryu and Rhoda M Alani
}

Melanoma is among the most virulent cancers, owing to its propensity to metastasize and its resistance to current therapies. The treatment failure is largely attributed to tumor heterogeneity, particularly subpopulations possessing stem cell-like properties, ie, melanoma stem-like cells (MSLCs). Evidence indicates that the MSLC phenotype is malleable and may be acquired by non-MSLCs through phenotypic switching upon appropriate stimuli, the so-called 'dynamic stemness'. Since the phenotypic characteristics and functional integrity of MSLCs depend on their vascular niche, using a two-dimensional (2D) melanoma-endothelium co-culture model, where the MSLC niche is recapitulated in vitro, we identified Notch3 signaling pathway as a micro-environmental cue governing MSLC phenotypic plasticity via pathwayspecific gene expression arrays. Accordingly, lentiviral shRNA-mediated Notch3 knockdown (KD) in melanoma cell lines exhibiting high levels of endogenous Notch3 led to retarded/abolished tumorigenicity in vivo through both depleting MSLC fractions, evinced by MSLC marker downregulation (eg, CD133 and CD271); and impeding the MSLC niche, corroborated by the attenuated tumor angiogenesis as well as vasculogenic mimicry. In contrast, Notch3 KD affected neither tumor growth nor MSLC subsets in a melanoma cell line with relatively low endogenous Notch3 expression. Thus, Notch3 signaling may facilitate MSLC plasticity and niche morphogenesis in a cell context-dependent manner. Our findings illustrate Notch3 as a molecular switch driving melanoma heterogeneity, and provide the biological rationale for Notch inhibition as a promising therapeutic option.

Laboratory Investigation (2017) 97, 725-736; doi:10.1038/labinvest.2017.1; published online 6 February 2017

Despite improved public education and increased awareness of the danger of sun exposure, the incidence of cutaneous melanoma continues to be on the rise for the past 30 years. Although successful public health campaigns have resulted in early detection and treatment, a significant portion of patients still succumb to metastatic disease, for which no current clinical regimen has proven significant long-term benefits. Melanoma cells are phenotypically and functionally heterogeneous, which is attributed to subpopulations of cells with stem cell-like properties, also known as melanoma stem-like cells (MSLCs). ${ }^{1}$ Recent studies have identified and characterized various MSLC subpopulations (including the $\mathrm{CD} 133^{+}$, $\mathrm{ABCB}^{+}, \mathrm{CD}_{271^{+}}$, $\mathrm{ALDH}_{1 \mathrm{~A}}^{+}, \mathrm{CD} 20^{+}$, and JARID1B ${ }^{+}$subsets). ${ }^{2-4}$ MSLCs exhibit increased resistance to conventional as well as state-of-the-art pathway-specific targeted therapies, ${ }^{5}$ and are responsible for clinical relapses, partly owing to their quiescence, upregulated efflux transporters, and heightened ability for DNA damage repair. ${ }^{6}$ Effective targeting of MSLCs, therefore, represents a crucial step to achieve a cure for melanoma. However, given the diverse MSLC subsets and the notion of a modified 'hierarchical'/stochastic model of CSCs, which postulates that several different subpopulations of CSCs co-exist in a given tumor, yet a subset of these may be better suited to survive and proliferate in a given microenvironment, the development of successful all-inclusive targeting strategies may require detailed understanding of the potential lineage and functional relationships among various MSLC fractions. At present, whether the various MSLC markers label the same, similar, or distinct population (s), and whether the various MSLC fractions are hierarchically, spatially, or functionally related remain unclear. Adding to the complexity is the growing awareness of cancer stemlike cell (CSC) plasticity, connoting that progenitor marker-negative cells can evolve into CSCs through proper environment cues or accumulating genetic alterations. ${ }^{7-11}$ Such 'dynamic stemness' is further supported by recent 
a

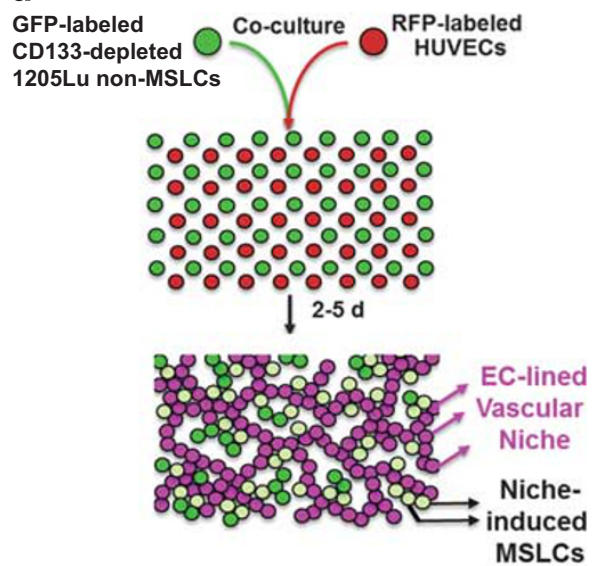

b

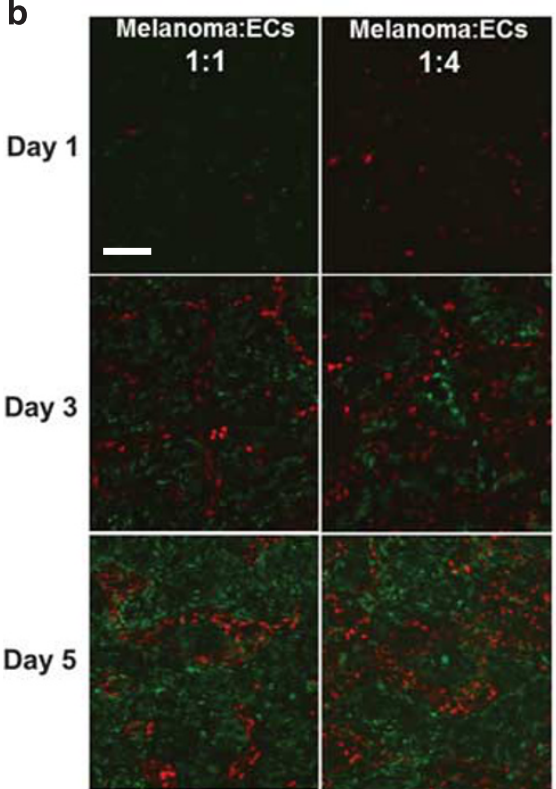

C

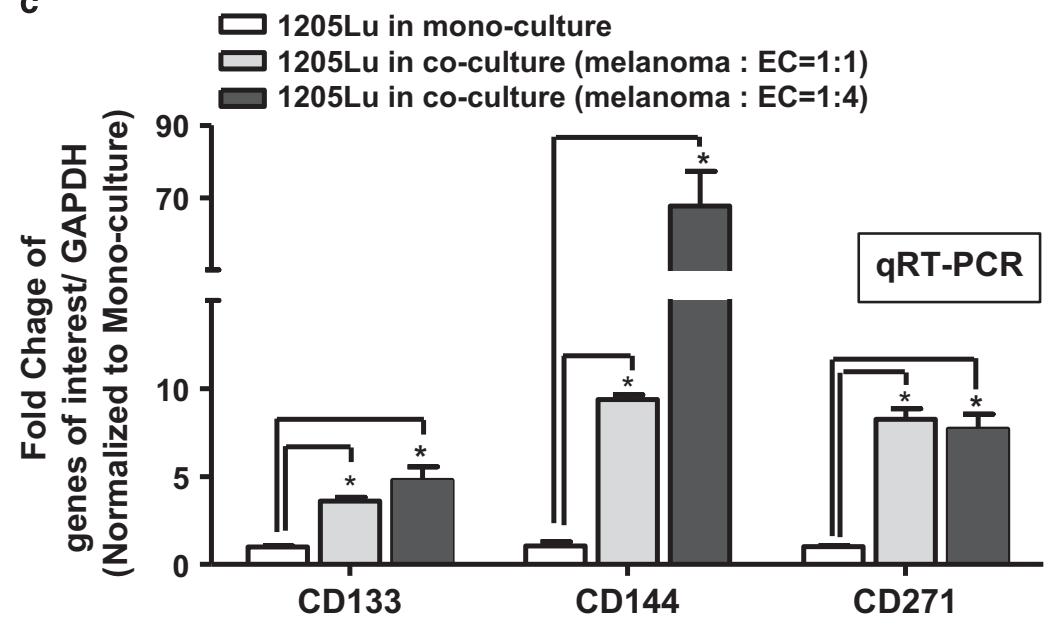

d

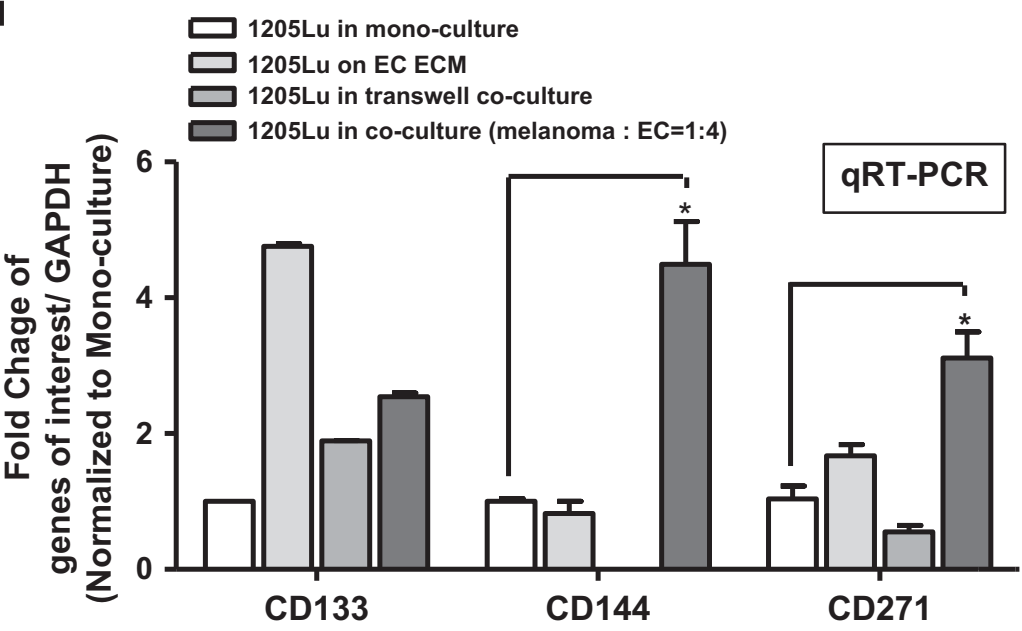

Figure 1 Two-dimensional (2D) melanoma-EC co-culture model recapitulates MSLC niche in vitro. (a) Schematic representation of the 2D MSLC niche model in vitro. GFP-labeled CD133- non-MSLCs were co-cultured with RFP-labeled human umbilical vein endothelial cells (HUVECs) at 1:1 or 1:4 ratios for 5 days. (b) In this model, ECs aligned to form branching tubular networks, reminiscent of the vascular niche in vivo (Magnification, $\times 100$; scale bar, $200 \mu \mathrm{m}$ ). Co-cultured melanoma cells were then segregated from ECs by flow cytometry. (c) MSLC (eg, CD133 and CD271) and VM (eg, CD144) markers were upregulated in co-cultured melanoma cells compared with their mono-culture counterparts using qRT-PCR, simulating 'dynamic stemness' and VM morphogenesis in vitro. (d) Such a niche-inducing phenomenon was most pronounced when melanoma-EC contact is permitted, while EC extracellular matrix (ECM) or soluble factors in transwell cultures alone exhibited limited/partial effects. ${ }^{*} P<0.05$.

reports where stem-like phenotype can be induced in nonCSCs in response to ectopic expression or upregulation of stem cell-associated transcription factors. ${ }^{12,13}$ Thus, targeting a specific class of cell surface markers may have limited benefit, as the CSCs are heterogeneous and genetically unstable. Ultimately, the success of CSC-targeted therapy may rely on niche-dependent strategies, since the phenotypic characteristics and functional integrity of CSCs depend on their existence within their niche. ${ }^{14-18}$

We have recently demonstrated that various MSLCs, ie, $\mathrm{CD}_{133^{+}}, \mathrm{CD} 271^{+}$, and $\mathrm{ABCB} 5^{+}$reside in a complex vascular niche, encompassing CD144/VE-cadherin ${ }^{+}$melanoma-lined vasculogenic mimicry (VM) channels, mosaic vessels, and authentic endothelial cell (EC)-lined blood vessels. ${ }^{7} \mathrm{VM}$, originally observed in uveal melanoma (and later in cutaneous melanoma and a myriad of human solid cancers), ${ }^{19}$ connotes the periodic acid-Schiff (PAS)-positive, perfusable patterned networks, and channels lined by aggressive tumor cells with endothelial phenotype, such as CD144 (VE-cadherin), Tie-1, and Eph2 expression. ${ }^{20-22}$ Expression gene profiling using microarray analyses reveals that tumor cells capable of VM exhibit a pluripotent, primitive phenotype, suggesting that the VM-engaging tumor cells possess stem cell-like properties with phenotypic plasticity to serve a stromal function. ${ }^{22}$ 
Given the functional co-dependence between the CSCs and their niche, we hypothesize that reciprocal signaling between MSLCs and their vascular niche may govern niche morphogenesis and stem cell homeostasis. However, the underpinning of such a crosstalk remains, by and large, unknown because of the lack of easily manipulable in vitro models that take into account the relevant and pivotal role of the niche environment. To elucidate the critical signaling pathways governing niche microenvironment support of tumor heterogeneity, we developed a simple $2 \mathrm{D}$ coculture system of melanoma cells and ECs that simulates the MSLC niche, where the MSLC phenotypic switch as well as vascular/VM niche morphogenesis are recapitulated in vitro (Figure 1). Using pathway-specific expression analyses, we identified Notch3 as a candidate that directs 'dynamic stemness' and niche morphogenesis. Targeting common niche signals controlling 'stemness', such as Notch3, represents a novel strategy to eliminate the diverse subsets of pre-existing MSLCs, as well as, the dynamically induced MSLC fractions that may evolve over time. The availability of existing Notch inhibitors currently used for Alzheimer's disease and many others emerging in the pharmaceutical market makes Notch inhibition a promising, fast-tracked therapeutic option for melanoma.

In human, the Notch pathway consists of four different transmembrane receptors, Notch1-4, and their membranebound ligands, Jagged (Jag1/2) and Delta (Dll1/3/4). Upon ligand binding, sequential proteolytic events, including cleavage by $\gamma$-secretase, release the active Notch intracellular domains (NICDs), which then translocate to the nucleus leading to transcriptional activation of the downstream Hes and Hey gene families. ${ }^{23}$ Overexpression of all four Notch receptors during melanoma progression has been reported. ${ }^{23}$ Although the oncogenic functions of Notch1 have been well documented, ${ }^{23}$ the roles of the other Notch paralogs remain largely unexplored. Only recently Hardy et al ${ }^{24}$ reported that Notch4 promotes melanoma aggressiveness, including VM and anchorage-independent growth, through Nodal, an embryonic morphogen of the TGF- $\beta$ superfamily implicated in the maintenance of stem cells.

Consistent with this, global $\gamma$-secretase inhibitors (GSIs) resulted in melanoma regression through Noxa-mediated apoptosis. $^{25,26}$ In another study, Howard et al ${ }^{27}$ identified Notch3 as one of the key mediators of melanoma-EC communication in a co-culture system, whose expression correlates with tumor progression. These findings corroborate with our hypothesis that Notch3-mediated melanoma-EC crosstalk regulates MSLC homeostasis and niche morphogenesis. To test our hypothesis, we used a lentiviral shRNAmediated loss-of-function approach using three independent melanoma cell lines with varying endogenous Notch3 levels in the context of MSLC niche in vitro and in vivo.

\section{MATERIALS AND METHODS Cell Culture}

Isogenic melanoma cell lines derived from different disease stages of tumor progression (obtained from Dr M. Herlyn at the Wistar Institute, Philadelphia, PA, USA) were cultured as previously described. ${ }^{28}$ These comprised primary vertical growth phase (VGP) melanoma cell lines as well as their metastatic isogenic counterparts, isolated from the same patient as the disease progressed. Red fluorescence protein (RFP)-labeled human umbilical vein endothelial cells (HUVEC) ${ }^{27}$ were obtained as a generous gift from Dr Rhoda Alani (Boston University, Boston, MA, USA) and cultured in EGM-2 (Lonza, Basel, Switzerland).

\section{In Vitro 2D Melanoma-Endothelium Co-Culture System, Recapitulating MSLC Niche}

Green fluorescence protein (GFP)-labeled 1205Lu melanoma cells $^{7}$ were depleted of CD133 ${ }^{+}$MSLCs using magnetic cell sorting (MACS) technology according to the manufacturer's protocol (Miltenyi Biotec, Bergisch Gladbach, Germany). $\mathrm{CD} 3^{-}$GFP-labeled 1205Lu melanoma cells and RFPlabeled HUVEC cells were plated at $~ 30 \%$ confluence at 1:1 or 1:4 ratios in EGM-2 culture medium. The cells were incubated for 5 days before segregating into pure populations (GFP vs RFP), using fluorescence activated cell sorting (FACS). Control mono-cultures were grown under identical conditions. RNA samples were prepared and subjected to the Stem Cell and Notch Signaling PCR Arrays based on the RT2 Profiler PCR Array User Manual (SA Biosciences/Qiagen, Valencia, CA, USA).

\section{Lentiviral Constructs and Infection}

To generate stable Notch3 knockdown (KD) cell lines using lentiviral vector, Notch3 shRNA and control lentiviral particles were generated in HEK293T cells by co-transfecting Notch3 shRNA or scrambled shRNA plasmids (Mission shRNA, Sigma-Aldrich, St Louis, MO, USA) and lentiviral packaging mix (Sigma-Aldrich) using Lipofectamine 2000 (Invitrogen, Waltham, MA, USA) according to the manufacturer's instruction. Notch3 stable KD cell lines were achieved by infecting cells with lentiviral particles and followed by selection in puromycin-containing medium ( $1 \mu \mathrm{g} / \mathrm{ml}$ for $1205 \mathrm{Lu} ; 2 \mu \mathrm{g} / \mathrm{ml}$ for A375 and WM852).

\section{Western Blotting}

Cells lysates or xenograft tissue homogenates were extracted in RIPA buffer (ThermoFisher Scientific) containing protease inhibitors cocktail (Roche, Basel, Switzerland) and quantified using a BCA protein assay kit (Pierce/ThermoFisher Scientific). Equal amount of proteins were subjected to electrophoresis in $4-12 \%$ SDS-PAGE gel (Invitrogen) and transferred onto nitrocellulose membranes. The blots were probed with anti-Notch3 (Cell Signaling Technology, D1118 rabbit mAb, Danvers, MA, USA), anti-CD133 (Miltenyi Biotech, clone W6B3C1), anti-CD271 (Alomone Labs, Jerusalem, Israel), anti-CD144 (Cell Signaling Technology) Abs. Beta-actin or tubulin (Abcam, Cambridge, UK) were used as a loading control. Densitometry analysis was 
performed using ImageJ software (http://rsb.info.nih.gov/ij/ download.html).

\section{Quantitative Real-Time Polymerase Chain Reaction}

RNA from melanoma cells or frozen tumor xenografts was extracted using an RNAeasy kit (Qiagen) and reversely transcribed using the SuperScript III RT cDNA Synthesis kit (Invitrogen) according to the manufacturer's protocol. Realtime quantitative PCR (qRT-PCR) was performed subsequently on a StepOnePlus Real-Time PCR System (Applied Biosystems/ThermoFisher) using SYBR select master mix (Applied Biosystems/ThermoFisher) and specific primers for CD133, CD144, CD271, and GAPDH. The primer sequences were as follows:

\section{CD133: forward 5'-TTCTTGACCGACTGAGAC-3'; reverse $5^{\prime}$-CCAAGCACAGAGGGTCAT-3'; \\ CD144: forward 5'-ATATGTCAGTGATGACTA-3'; reverse $5^{\prime}$-CTTACCAGGGCGTTCAGG-3'; \\ CD271: forward 5'-ACTCACTGCACAGACTCT-3'; reverse 5'-GAAGCTTCTCAACGGCTC-3' \\ GAPDH: forward 5'-CGACAGTCAGCCGCATCTT-3'; reverse 5'-ACCTTCCCCATGGTGTCTCA-3'}

All the samples were run in triplicate and normalized to the housekeeping gene, GAPDH. Data were analyzed using the $2^{-\Delta \Delta \mathrm{Ct}}$ method.

\section{Anchorage-Dependent (Growth Curve) and -Independent (Soft Agar) Growth Assays}

For growth curve assay, the cells were plated at $1 \times 10^{4} /$ well in six-well plates and counted after 3, 7, and 10 days using hemocytometer. Soft agar assay was performed as previously described. ${ }^{29}$ Briefly, the cells were suspended in MCDB153/ L15 medium (v/v: 4/1) supplemented with $25 \mu \mathrm{g} / \mathrm{ml}$ bovine extract, $2 \mathrm{ng} / \mathrm{ml}$ epidermal growth factor (EGF), $2 \mu \mathrm{g} / \mathrm{ml}$ insulin, $4 \%$ fetal bovine serum, and $0.25 \%$ agar and plated in triplicate at $1 \times 10^{4}$ cells per well in six-well plates. After 2 weeks, colonies with more than five cells were counted in 20 randomly chosen fields $(\times 100)$ using an inverted microscope and the percentage of colony formation was calculated. Each experiment was repeated twice with consistency. The represented data were compiled from the independent repeats, and the data were analyzed using the Student's $t$-test.

\section{In Vitro Tube-Forming Assay}

The $50 \mu$ Matrigel (BD Biosciences, San Jose, CA, USA) was plated in 96-well culture dishes and allowed to polymerize at $37^{\circ} \mathrm{C}$ for $30 \mathrm{~min}$. A total $4 \times 10^{4}$ cells per well were then seeded on the Matrigel layer and grown for $24 \mathrm{~h}$. Randomized fields were captured using inverted microscope (Nikon, Tokyo, Japan). For quantitative analysis of tube formation and length, three randomly selected microscopy fields $(\times 200)$ were photographed per experimental condition. Tube formation was analyzed by ImageJ software, and the data were analyzed using the Student's $t$-test.

\section{Immunofluorescence}

Frozen melanoma xenograft sections were subjected to double immunofluorescence using standard procedures. ${ }^{29}$ The primary antibodies used in this study are rabbit antihCD144 (human-specific; Cell Signaling Technology), rabbit anti-hCD133 (human-specific; Miltenyi Biotech), rabbit antihCD271 (human-specific; Alomone Labs), and rat antimCD31 (mouse-specific; BD Biosciences). The secondary antibodies used are FITC-conjugated donkey anti-rabbit IgG, AlexaFluor 594-conjugated donkey anti-rat IgG, and AlexaFluor 488-conjugated goat anti-mouse IgG1 (Invitrogen) antibodies. Isotype-matched rabbit, mouse, or rat immunoglobulin was used in place of the primary antibody for control. The sections were mounted with VectaShield containing DAPI (Vector Laboratories, Burlingame, CA, USA) and examined under a Nikon Eclipse E400 microscope equipped with FITC and TRITC filters (Nikon) and a Mercury-100W lamp (Chiu Technical Corporation). Host angiogenesis $\left(\mathrm{mCD} 1^{+}\right)$was quantified by measuring luminal area or number of tubules per field $(\times 40)$ using ImageJ software, and the data were analyzed using the Student's $t$-test. Quantification of human melanoma-derived VM channels was performed by measuring the $\mathrm{hCD} 144^{+}$area per field $(\times 100)$ using ImageJ software, and the data were analyzed using the Student's $t$-test.

\section{In Vivo Tumorigenicity}

The mice are maintained under pathogen-free conditions in an American Association for Accreditation of Laboratory Animal Care-accredited facility at the Boston University Medical Center under the supervision of the Laboratory Animal Science Center and its staff of veterinarians and support personnel. For the effect of Notch3 silencing on tumorigenicity, $2 \times 10^{6} 1205 \mathrm{Lu}$, A375, or $4 \times 10^{6}$ WM852 cells infected by control and Notch3 shRNA lentiviral constructs were injected subcutaneously in the dorsal skin of each severe combined immune-deficient (SCID) mouse (CB17; Taconic Laboratory; four to five mice per condition). The tumor growth was monitored twice per week after implantation. The tumor dimensions were measured using calipers and the tumor volume was calculated as previously described. ${ }^{29}$ Melanoma xenografts were harvested when the tumors reached approximately $1 \mathrm{~cm}^{3}$, and subjected to further analyses, including immunofluorescence, qRT-PCR, and western blotting.

\section{Statistical Analysis}

Differences between two groups were analyzed using the Student's $t$-test. Differences between more than two groups were analyzed by one-way ANOVA. Two-sided $P$-values less than 0.05 were considered significant. 


\section{RESULTS}

Upregulation of Notch3 Expression in the 2D MSLC Niche Model In Vitro

To study MSLC biology in a tissue context, we developed a simple 2D co-culture system, stimulating the MSLC niche. In this model, GFP-labeled, CD133-depleted, non-MSLC 1205Lu melanoma cells, and RFP-labeled human umbilical vein endothelial cells (HUVECs) were mixed at two different ratios (1:1 vs 1:4), and co-cultured for 5 days (Figure 1a). Over time, the endothelial cells aligned themselves to form interconnecting channels/networks around the melanoma cells, mimicking the MSLC vascular niche (Figure 1b). As a consequence, the expression of stem-like cell markers, CD133 and CD271, as well as VM marker, CD144, increased dramatically in sorted melanoma cells from the 2D co-culture system compared with their mono-cultured counterparts (Figure 1c). These findings demonstrate that the 2D co-culture system recapitulates MSLC niche morphogenesis in vitro. To determine whether MSLC niche induction in vitro is mediated by direct cell-cell contact, extracellular matrix (ECM) or soluble factors secreted by HUVECs in our 2D MSLC model, we examined stem-like cell (ie, CD133 and CD271) and VM (ie, CD144) marker expression by qRT-PCR additionally in melanoma cells conditioned by HUVEC ECM and melanoma cells collected from transwell coculture with HUVECs. We found that the expression of CD133, CD271, and CD144 was most consistently induced when melanoma-HUVEC contact was permitted (Figure 1d).

To identify molecular signals driving niche morphogenesis and MSLC induction, flow cytometry-segregated $1205 \mathrm{Lu}$ melanoma cells and HUVECs from the 2D MSLC model (mono-cultured counterparts as control; Figure 2a) were subjected to gene expression profiling using stem cell and notch signaling pathway-specific PCR arrays (SA Biosciences/ Qiagen). We found that Notch3 is concurrently upregulated in both co-cultured melanoma cells and HUVECs from the 2D MSLC model (Figure 2a). Interestingly, Notch downstream signaling mediators, Hes1 and Heyl, were concomitantly elevated in co-cultured melanoma cells, suggesting the activation of the Notch pathway (Figure 2a). These results were verified using qRT-PCR (Figure $2 \mathrm{~b}$ and data not shown). Similar to MSLC and VM markers induction, Notch3 expression was highly increased when melanoma-HUVEC contact was permitted, while HUVEC ECM and secretory soluble factors conveyed partial effects (Figure 2c). In addition, Notch4 and Jag1, and Dll1 were also increased in co-cultured melanoma cells, and ECs, respectively. Taking together, our findings corroborate with the hypothesis that the Notch (particularly Notch3)-mediated cell-cell contact between the melanoma cells and endothelial cells may contribute to the induction of MSLC phenotype and niche morphogenesis.

\section{Specific and Functional Notch3 Silencing in Melanoma Using the Lentiviral shRNA Approach}

To examine the role of Notch3 in MSLC niche biology, we screened endogenous Notch3 expression in a panel of 11 melanoma cell lines from different stages of tumor progression using western blotting (Figure 3a). On the basis of this screening, most cell lines exhibited low endogenous level of Notch3. Two metastatic melanoma cell lines $1205 \mathrm{Lu}$ and WM852 show high endogenous expression of both full-length Notch3 and the active intracellular domain forms (Figure 3a). Among these cell lines, we chose high Notch3-expressing cell lines, 1205Lu and WM852, as well as one relatively low Notch3-expressing cell line, A375, to explore the functional role of Notch3 with regard to tumor growth, and niche morphogenesis/homeostasis. We generated stable Notch3 KD cells using lentivirus-based shRNAs (Mission shRNA, SigmaAldrich). Western blot analyses confirmed Notch3 KD at the protein level in vitro compared with control cells expressing non-target shRNA (Figure 3b). Quantitative RT-PCR verified downregulation of Notch3 message levels in vitro (Supplementary Figure S1A). As members of the Notch gene family are highly homologous, to determine whether Notch3 silencing is specific and functional, we examined the expression of other Notch genes and well-known Notch3 targets, Hes1 and Hes2, using qRT-PCR. Despite slight, statistically nonsignificant reduction of Notch1 and 4 in WM852 Notch3 shRNA2 cells, no significant nonspecific change in other Notch paralogs was noted in all three Notch3 KD cells (Supplementary Figure S1B). Notch3 targets, Hes1 and Hes2, were both downregulated in 1205Lu and WM852 Notch3 KD cells, but in A375 Notch3 KD cells, only Hes2, but not Hes1, was downregulated (Supplementary Figure S1C). These data demonstrated the achievement of specific and functional silencing of Notch3 in melanoma cells.

\section{Biological Consequences of Notch3 KD In Vitro, Including Cell Growth, Colony-Forming Ability, VM/Tube Formation on Matrigel, and MSLC Niche Induction in 2D Niche Model}

To explore the effect of Notch3 KD on cell proliferation in vitro, we performed conventional as well as anchorageindependent growth assays, using non-target shRNA transduced cells as controls. Notch3 KD in 1205Lu and A375 cells did not modulate cell growth or colony-forming ability (Figure $4 \mathrm{a}$ and $\mathrm{b}$, left and middle panels). In contrast, Notch3 KD led to a significant inhibition in WM852 cell growth as well as colony-forming ability (Figure $4 \mathrm{a}$ and $\mathrm{b}$, right panel). In addition, Notch3 KD in $1205 \mathrm{Lu}$ cells resulted in a significant attenuation in VM tubule formation on Matrigel, as measured by the number of branch point/field $(\times 200$; Figure 4c). Both non-target control and Notch3 KD A375 or WM852 cells were unable to form VM tubules in the Matrigel tube-formation assays (data not shown). These results demonstrated that Notch3 KD exhibits variable cell linespecific inhibitory effects on growth, colony formation, and $\mathrm{VM} /$ tubule formation in vitro.

To determine whether Notch3 KD affects niche morphogenesis in vitro, we incorporated GFP-labeled 1205Lu Notch3 $\mathrm{KD}$ cells and RFP-labeled HUVEC cells in our 2D co-culture 

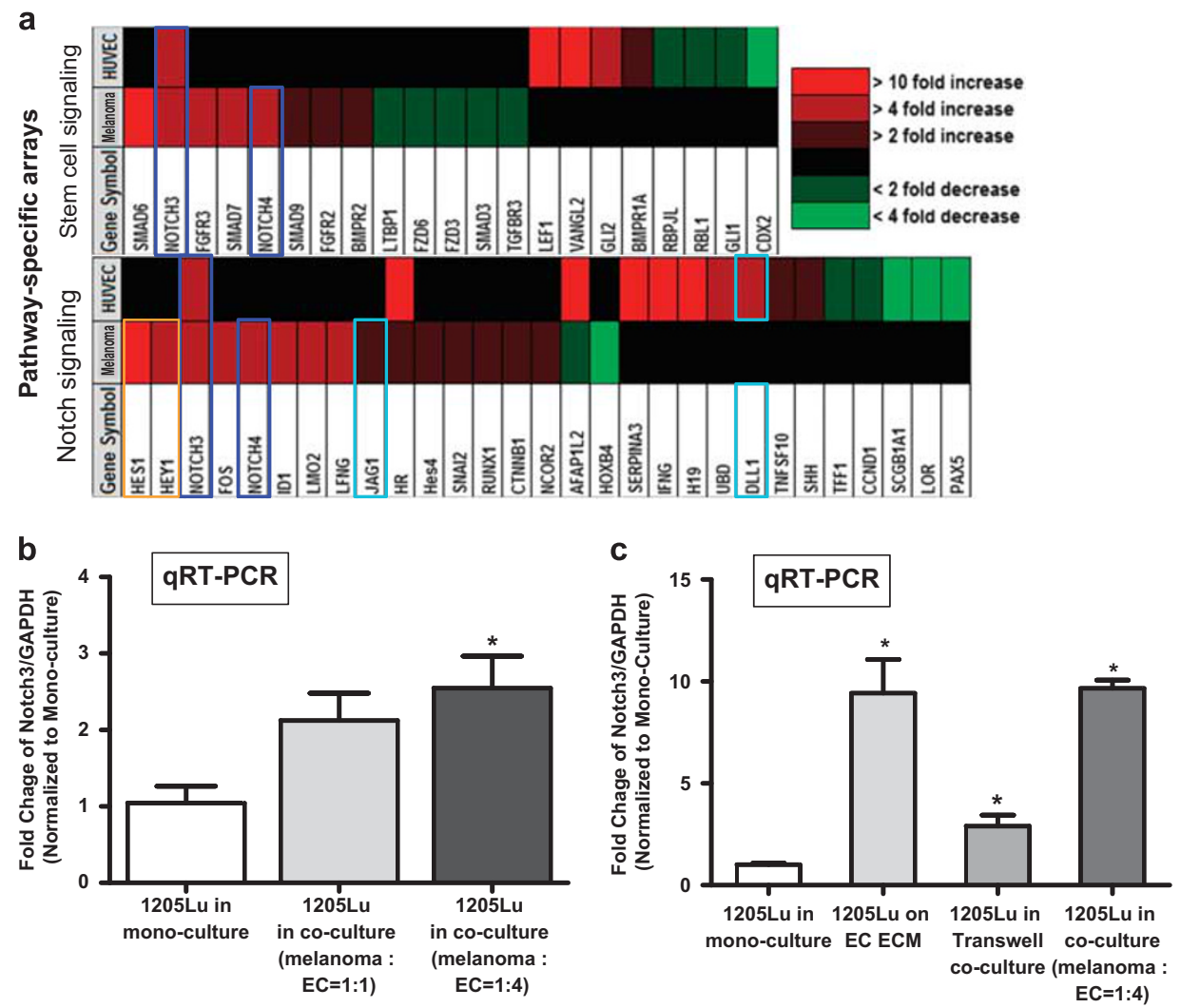

Figure 2 Notch3 upregulation in co-cultured melanoma cells and ECs in the 2D niche model. (a) Flow cytometry-segregated melanoma cells and ECs from the 2D niche model were subjected to stem cell and notch signaling pathway-specific PCR arrays (SA Biosciences) using their mono-culture counterparts as control. Notch3 was consistently upregulated both in co-cultured melanoma cells and ECs. The concomitant upregulation of Notch downstream effectors, Hes1 and Hey1, further confirmed activated Notch signaling in co-cultured melanoma cells. (b) qRT-PCR validated Notch3 induction in co-cultured melanoma cells following sorting by flow cytometry. The data were expressed as fold change of the ratio between Notch 3 and GAPDH mRNA in co-cultured melanoma cells, normalized to that of their mono-culture counterpart. (c) The magnitude of Notch3 induction in co-cultured melanoma cells was most evident when direct cell-cell contact is allowed, while EC ECM or soluble factors in transwell co-cultures produced only limited/partial result. ${ }^{*} P<0.05$.

niche model, and then measured CD133, CD271, and CD144 message levels using qRT-PCR following flow cytometrysorting (Figure 4d). Consistent with our hypothesis that Notch3 mediates stem cell phenotype and vascular niche formation, co-culture with HUVECs failed to induce stem cell (eg, CD133 and CD271), as well as VM (eg, CD144) marker expression in Notch3 KD 1205Lu cells, while significant upregulation of these markers was observed in co-cultured non-target control cells. These results highlight a pivotal role of Notch3 in MSLC niche induction and homeostasis.

\section{Notch3 KD Retards Tumor Growth by Depleting MSLCs and Compromising Vascular Niche In Vivo}

Given that 1205Lu Notch3 KD shRNA \#2, A375 Notch3 KD shRNA \#1, and WM852 Notch3 KD shRNA\#2 cells exhibited greater Notch3 KD efficiencies (Figure 3b), we chose to use these cells for tumorigenicity studies. In 1205Lu and WM852 cells, which have high endogenous Notch3 expression, Notch3 KD significantly retarded xenograft growth compared with the non-target control (Figure 5a and c). Although growth was delayed, 1205Lu Notch3 KD xenografts did eventually develop tumors. On the other hand, Notch3 KD completely abolished tumor growth in WM852 cells as no tumor was observed 14 weeks post inoculation despite high numbers of cells $\left(4 \times 10^{6}\right.$ cells $)$ injected. In contrast, tumor growth of A375, a cell line that exhibits relatively low endogenous Notch3 expression, was not significantly affected by Notch3 KD (Figure 5b). These findings suggest that Notch3 KD attenuates tumor growth in vivo and the growth regulatory effect of Notch3 depends on the cellular context relevant to its level of endogenous expression. Effective Notch3 KD in 1205Lu and A375 melanoma xenografts was confirmed both at the mRNA (data not shown) and protein (Figure 6a and c) levels. Because no tumor xenografts developed from Notch3 KD WM852 cells following 14 weeks of extended observation despite injection of high numbers of cells $\left(4 \times 10^{6}\right.$ cells/mouse), at which time the control tumors grew larger than $1 \mathrm{~cm}^{3}$ and the experiment had to be terminated, further analyses of WM852 xenografts could not be performed. 
a

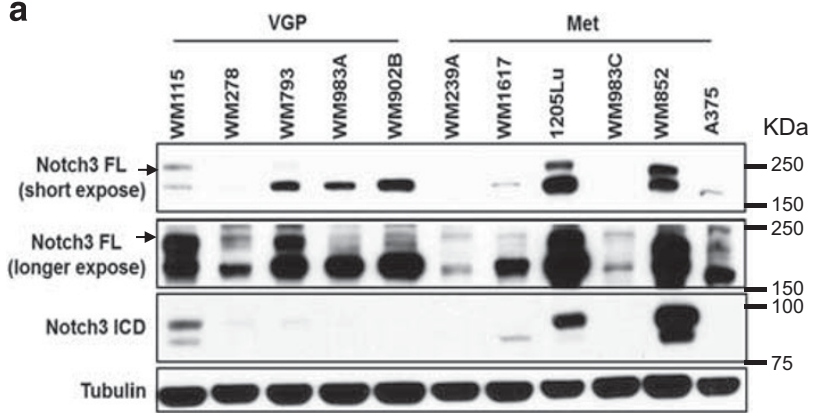

b

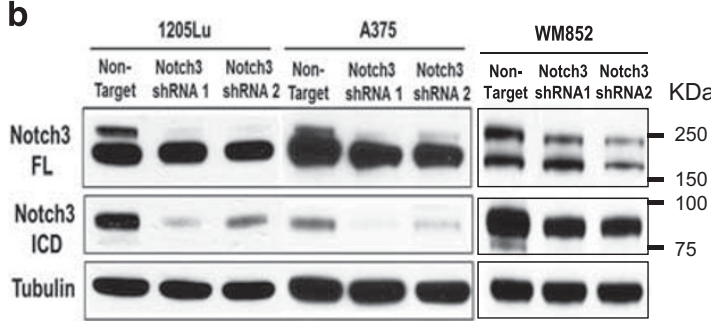

Figure 3 Notch3 silencing in melanoma cells using lentiviral shRNA. (a) Multiple melanoma cell lines of different stages of tumor progression (ie, vertical growth phase (VGP) and metastasis (Met)) expressed endogenous Notch3 by western blotting. Tubulin was used as a loading control. (b) Using lentiviral shRNA (non-target shRNA as control), significant KD efficiency was achieved in 1205Lu, A375 and WM852 melanoma cell lines as shown by western blotting.

To explore the biological significance of Notch3 on niche morphogenesis in vivo, we analyzed tumor vasculatures in human melanoma xenografts using multi-label immunofluorescence (IF; Figure 5d). Parallel to the data obtained from in vitro tubule/VM formation analyses, CD $144^{+}$VM-like melanoma channel formation was significantly hindered in Notch3 KD 1205Lu xenografts compared with their control counterparts (Figure 5d), quantified by the area of CD144 channels (Figure 5f). Consistent with these, CD144 and Tie-1 (a VM-associated gene) expression was significantly downregulated in Notch3 KD 1205Lu xenografts compared with the control tumors (Figure $6 \mathrm{a}$ and $\mathrm{b}$ ). Since VM formation in the xenografts is intimately associated with authentic host vessels in keeping with perivascular niche, ${ }^{7,30}$ we examined the impact of Notch3 KD on in-grown mouse vessels within the melanoma xenografts. Accordingly, we found that host angiogenesis, characterized by $\mathrm{mCD} 31^{+}$blood vessels, was significantly reduced in Notch3 KD 1205Lu xenografts as measured by the $\mathrm{mCD} 31^{+}$luminal area and number of $\mathrm{m}$ $\mathrm{CD}^{+} 1^{+}$tubules (Figure 5e). These results demonstrate that Notch3 KD inhibits tumor angiogenesis as well as VM formation in vivo, both of which are integral components of the MSLC niche. To further analyze Notch3 KD on MSLC homeostasis, we examined expression of stem cell markers, CD133 and CD271, in xenografts by western blot analysis. We found that protein expression of CD133 and CD271 was reduced in Notch3 KD 1205Lu xenografts compared with their non-target control counterparts (Figure 6a), suggesting that Notch3 KD in 1205Lu melanoma cells leads to loss of stem-like cell phenotype in vivo. Consistent with the observed differential effect of Notch3 KD on tumorigenicity in A375 melanoma cells, the expression of stem-like cell marker, CD271, was not affected in Notch3 KD A375 xenografts (Figure 6c). Attempts to further validate CD133 and CD144 in Notch3 KD A375 xenografts using western blot analysis (C8161 lysate included as a positive control) were unsuccessful due to sensitivity issue (Figure 6c; note that CD133 and CD144 signals were undetectable both in the control and KD xenografts despite maximal loading and prolonged overnight exposure). Collectively, these data suggest that Notch3 KD may inhibit perivascular niche morphogenesis and MSLC homeostasis through impeding tumor angiogenesis and VM. However, such effects are cell context-dependent.

\section{DISCUSSION}

A major gap in MSLC research (or CSC research in general) is the lack of simple and easily reproducible in vitro models that takes into account the relevant and pivotal role of the niche microenvironment. As the existence in a supportive "niche ${ }^{7,30}$ is integral to the functional integrity of CSCs, studies on isolated CSCs without the 'niche' context are inherently suboptimal. Despite significant advances in mouse xenograft models that provide accessible platforms to study MSLC biology in the mouse host 'niche' environment, time and financial constraints as well as the individual host variability negatively impact on their routine use. To facilitate dissecting the niche signals responsible for MSLC 'stemness' as a gateway to develop all-encompassing targeting strategies for MSLCs, whether pre-existing or dynamically induced, we developed a robust and easily reproducible $2 \mathrm{D}$ in vitro co-culture model system that is simple enough to be manipulated yet sophisticated enough to reflect the complexities inherent to the niche environment. This melanomaendothelium co-culture niche model simulates MSLC niche, where the vascular/VM niche and 'dynamic stemness' are recapitulated. In this model, stem cell (CD133 and CD271) and VM (CD144/VE-cadherin) markers are induced in nonMSLCs upon co-culture with ECs (Figure 1). Using flow cytometry, various MSLC subsets can be isolated, permitting subsequent expression profiling to identify differentially expressed genes comparing with the non-MSLCs. In addition, this model allows manipulation of single or multiple variables sequentially or simultaneously (with the use of conventional and inducible vectors) in one or both cellular constituents, maximizing the ability to elucidate gene interactions in a defined system that preserves tissue context. More importantly, such a model can be easily adapted for other solid cancers, providing a robust and well-defined experimental system to study CSC-stromal interplay.

Given that Notch signaling-mediated cell-cell communication is known to have major roles in cell fate decisions, lineage specification, as well as stem cell homeostasis in multiple 
organ systems not only during embryonic development but also in adult life, ${ }^{31}$ it is not surprising that we identified Notch 3 as one of the key differentially expressed genes by the dynamically induced $\mathrm{CD}_{133^{+}}$MSLCs in the $2 \mathrm{D}$ niche coculture model using pathway-specific PCR arrays following segregation by flow cytometry. Accordingly, Notch3 KD in 1205Lu melanoma cell lines expressing high levels of endogenous Notch3 resulted in retarded/abolished tumorigenicity (Figure 5a), as well as, attenuated MSLC subsets both in the $2 \mathrm{D}$ niche co-culture system in vitro (Figure $4 \mathrm{~d}$ ) and in a

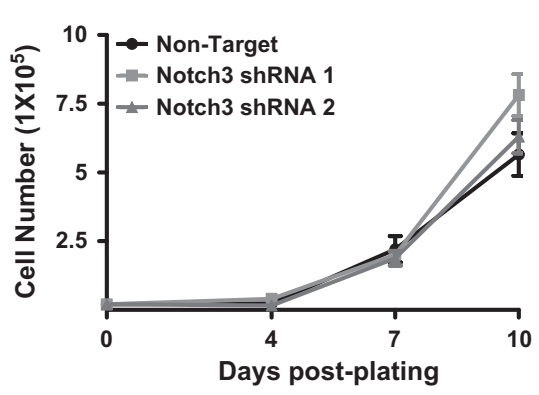

A375

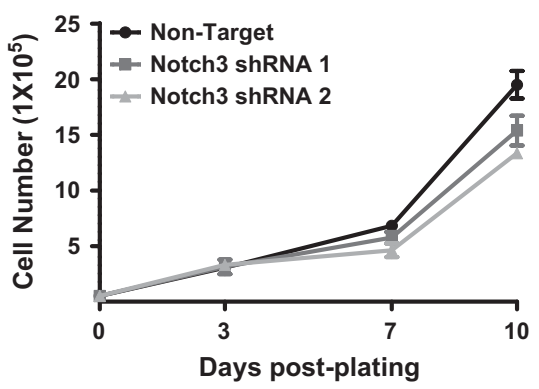

WM852

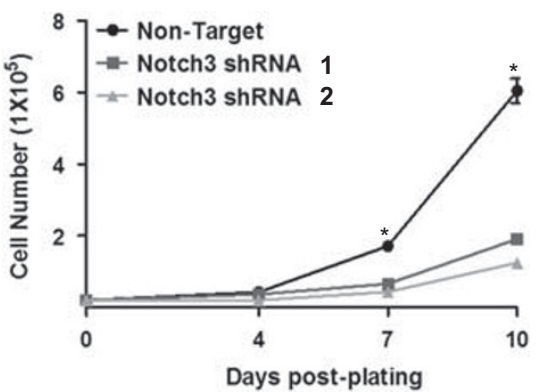

b
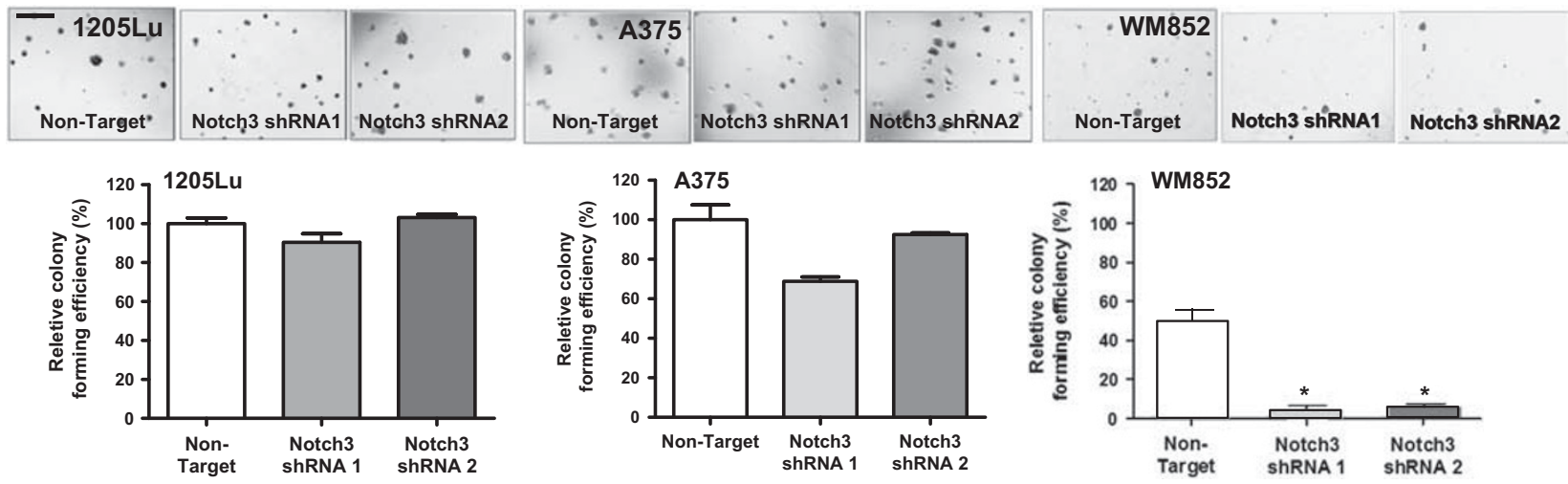

C
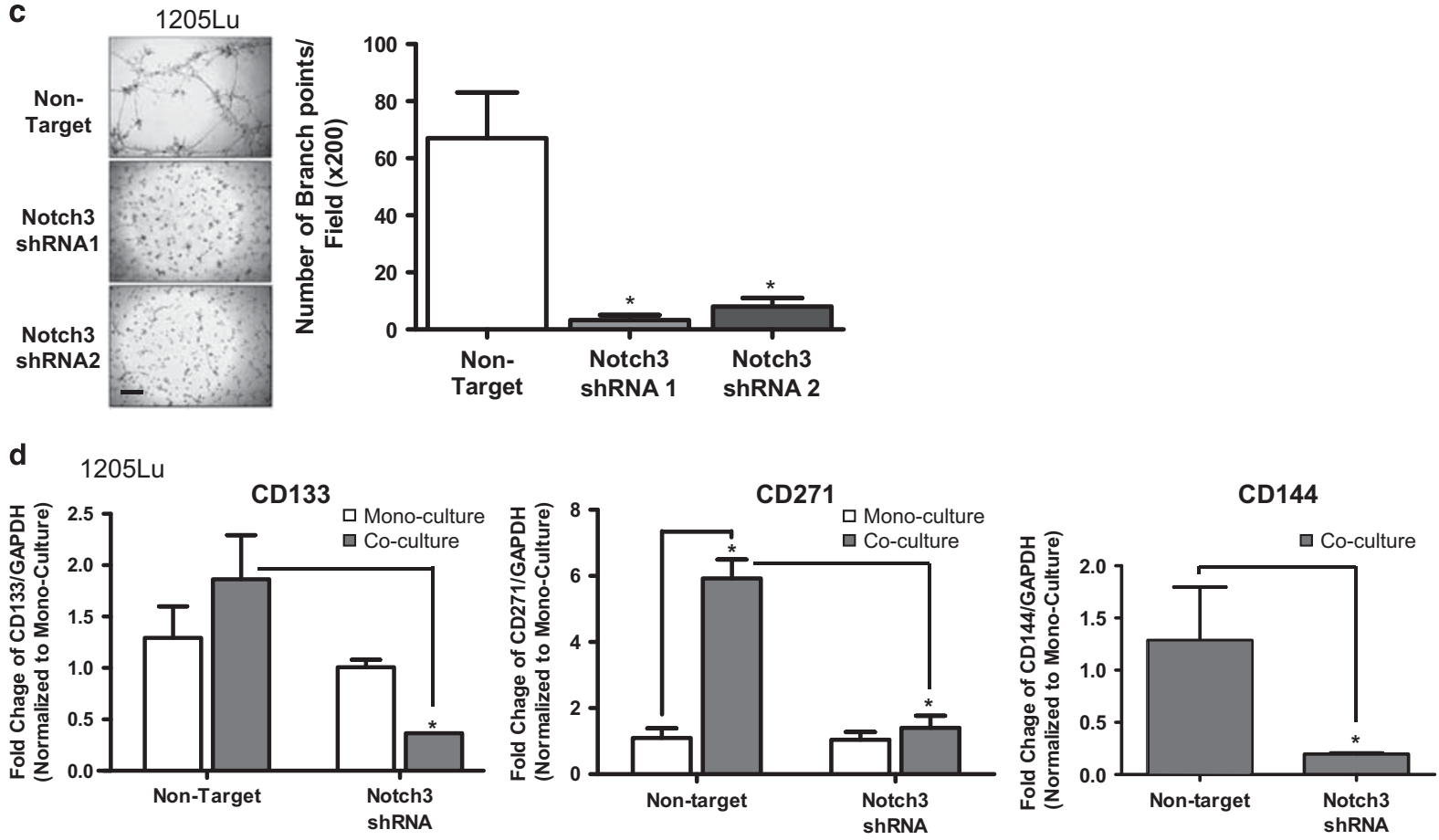
the xenograft model in vivo (Figure 6a). These data support a pivotal role of Notch3 in MSLC induction, maintenance, and homeostasis at least in a subset of melanomas. Parallel to our findings, a novel $\gamma$-secretase inhibitor (GSI), RO4929097, was shown to inhibit melanoma cell proliferation, colony-forming ability, and $3 \mathrm{D}$ spheroid growth in vitro, and to suppress a

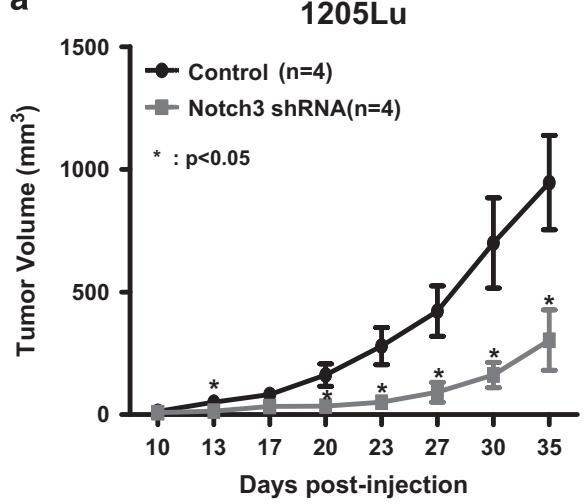

b

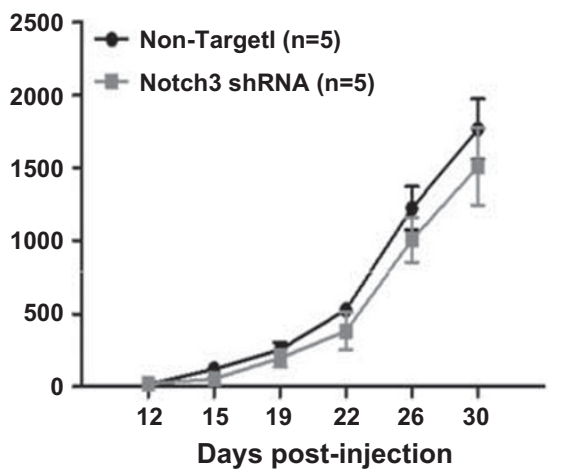

WM852

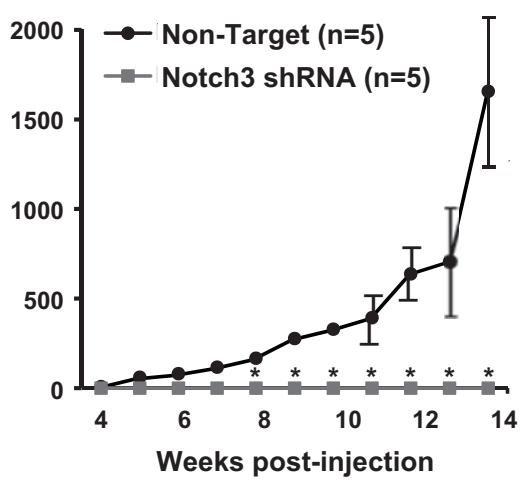

d
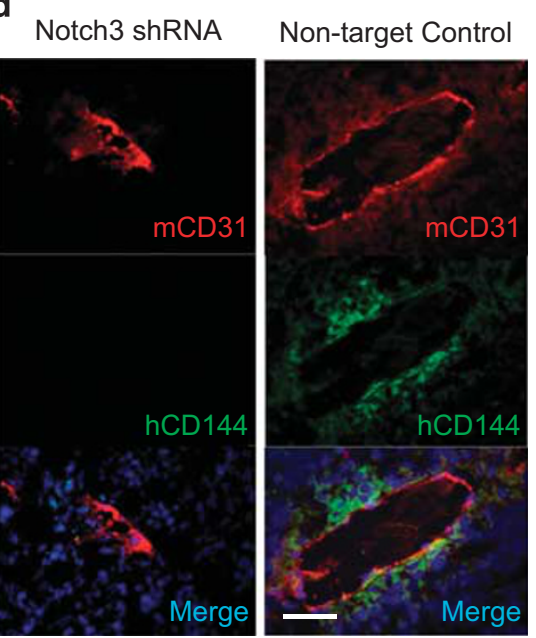

e
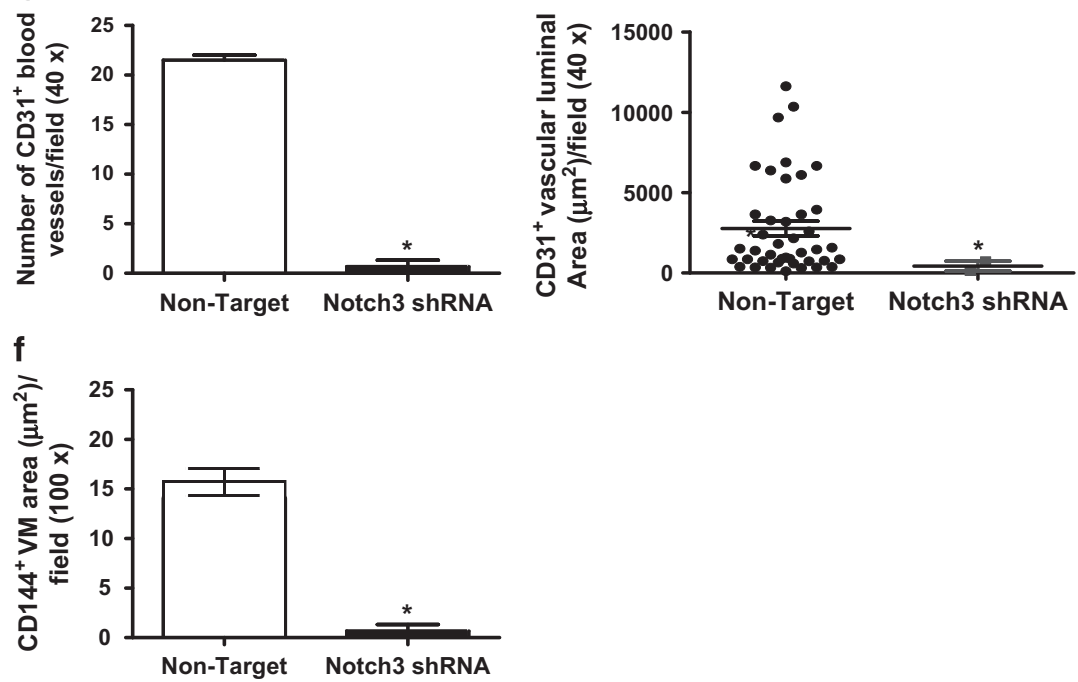

Figure 5 In vivo consequences of Notch3 KD in a melanoma xenograft model. In 1205Lu and WM852 melanoma cells, displaying high levels of endogenous Notch3, Notch3 KD retarded/abolished tumorigenicity (a and c). In contrast, in A375 cells, harboring relatively low endogenous Notch3 expression, Notch3 KD failed to affect tumor growth $(\mathbf{b})$. Analyses of the tumor samples using double immunofluorescence coupled with quantification using ImageJ software revealed that Notch3 KD inhibited tumor angiogenesis as well as melanoma-derived VM. Immunofluorescence analysis of $1205 \mathrm{Lu}$ xenograft sections double labeled for $\mathrm{mCD} 31^{+}$host blood vessels (red), and hCD $144^{+} \mathrm{VM}$ (green) showed that Notch3 KD xenografts displayed smaller and much less prominent $\mathrm{mCD} 31^{+}$host vessels and abolished VM (d, left panels) compared with non-target control xenografts (d, right panels); magnification, $\times 400$; scale bar, $50 \mu \mathrm{m}$. Human melanoma-derived VM vessels $\left(\mathrm{hCD} 144^{+}\right)$and host angiogenesis $\left(\mathrm{mCD} 31^{+}\right)$were quantified as measured by the average luminal area or number of tubules per $\times 40$ or $\times 100$ field and revealed a significant reduction in the size and number of mCD31 $1^{+}$host blood vessels (e), as well as hCD144+ VM area (f) in 1205Lu Notch3 KD xenografts compared with controls. Analyses of A375 xenografts failed to show significant difference in tumor angiogenesis and VM between Notch3 KD and control (data not shown). As tumor growth in WM852 cells was completely abolished, no KD xenografts were available for further analyses. ${ }^{*} P<0.05$.

Figure 4 In vitro consequences of Notch3 KD in melanoma. In 1205Lu melanoma cells, exhibiting high endogenous Notch3 (Figure 3a), Notch3 KD resulted in no effects on conventional 2D growth (a, left panel) or colony-forming ability in soft agar (b, left panels; magnification, $\times 100$; scale bar, $500 \mu \mathrm{m}$ ), but significantly inhibited VM in Matrigel tube-formation assays (c; $\times 200$, scale bar, $100 \mu \mathrm{m}$ ). When incorporated into the $2 \mathrm{D}$ niche co-culture model, Notch3 KD hindered EC-mediated MSLC induction and VM phenotypic switch in 1205Lu cells (d). Similarly, Notch3 KD in A375 cells, which demonstrated relatively low endogenous Notch3 expression (Figure 3a), showed no effect on conventional 2D growth (a, middle panel) or colonyforming efficiency (b, middle panels). In contrast, in another cell lines displaying high baseline Notch3 expression, WM852, Notch3 KD led to significant growth inhibition under conventional culture condition (a, right panel), as well as 3D anchorage-independent growth in soft agar assays (b, right panels). The effect of Notch3 KD in A375 and WM852 cells on VM in Matrigel tube-formation assays was assessed; however, both non-target control and Notch3 KD counterparts failed to undergo VM (data not shown). ${ }^{*} P<0.05$. 
a

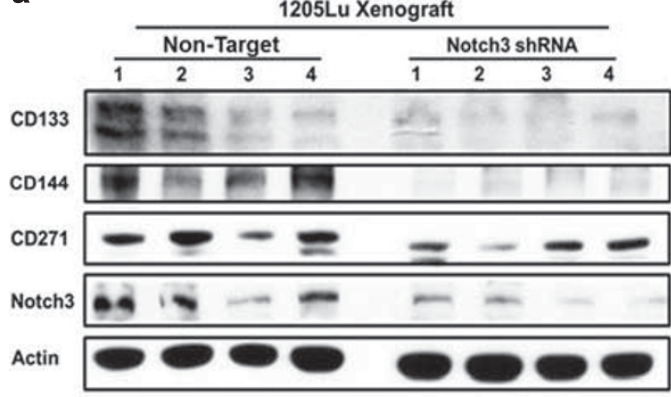

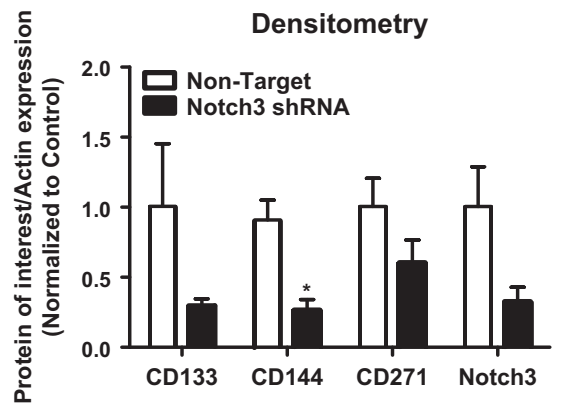

C

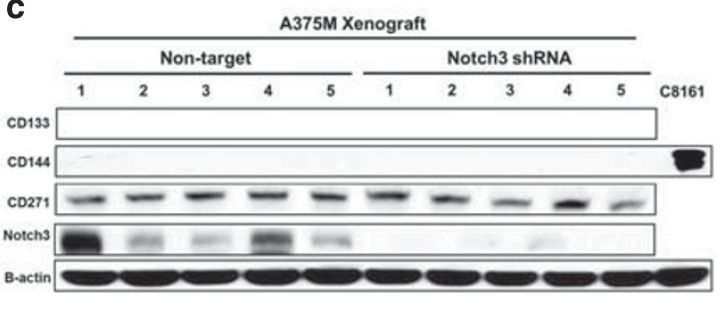

Figure 6 Effective and functional Notch3 KD depletes MSLCs and VM-engaging melanoma cells in 1205Lu xenografts. (a) Western blot analyses demonstrated that stem cell markers (eg, CD133 and CD271) as well as VM marker, CD144, were downregulated in Notch3 KD 1205Lu tumors, compared with non-target control (a). Consistent with the observed VM inhibition by Notch3 KD above, the expression of Tie-1, a VM-associated gene, was also downregulated in Notch3 KD 1205Lu xenografts by qRT-PCR (b). Of note, the efficacy of Notch3 KD was maintained in the tumor xenografts as shown by Western blotting (a). On the other hand, in A375 melanoma cells where no change in tumorigenicity was appreciated following Notch3 KD, the expression of stem-like cell marker, CD271, was not affected (c). Attempts to further validate CD133 and CD144 in Notch3 KD A375 xenografts using western blot analysis (C8161 lysate included as a positive control) were unsuccessful due to sensitivity issue (c; note that CD133 and CD144 signals were undetectable both in the control and KD xenografts despite maximal loading and prolonged overnight exposure). ${ }^{*} P<0.05$.

tumor-initiating potential in a serial xenotransplantation model. ${ }^{32}$ In addition, Notch3 signaling enriches CSCs of the lung, ${ }^{33}$ colorectal, ${ }^{34}$ hepatocellular, ${ }^{35}$ and breast ${ }^{36}$ cancers, and Notch2/Notch3 antagonists/dominant-negative receptors inhibit tumor growth and decreases tumor-initiating cell frequency in a broad spectrum of epithelial tumors, including breast, lung, ovarian, and pancreatic cancer. ${ }^{37}$

Derangements of Notch pathways are commonly observed in human cancers, including melanoma, ovarian, endothelial, cervical, lung, breast, renal, head and neck, prostate, mesothelioma, and various hematological malignancies. ${ }^{38}$ Although the oncogenic functions of Notch1 in melanoma have been well established, ${ }^{39,40}$ the roles of the other Notch receptors in melanoma remain substantially uncharted. Only recently, Hardy et al, ${ }^{24}$ reported that Notch4 promotes melanoma aggressiveness, including VM- and anchorageindependent growth through Nodal, an embryonic morphogen of the TGF- $\beta$ superfamily implicated in the maintenance of embryonic stem cells. Consistent with this, global Notch inhibition by $\gamma$-secretase inhibitors (GSIs) results in reduced tumor-initiating potential of melanoma in vitro in soft agar and spheroid-formation assays, as well as in vivo in a serial xenotransplantation model. ${ }^{32}$ In another study, GSI treatment leads to melanoma regression through Noxa-mediated apoptosis in mice. ${ }^{25}$ Recently, Howard et al, ${ }^{27,41}$ in a coculture system, identified Notch 3 as one of the key mediators of melanoma-EC communication, whose expression correlates with tumor progression, and activated Notch3 signaling results in accelerated melanoma cell migration without affecting tumor cell growth. These findings are in line with our observations that Notch3 KD in melanoma cell lines expressing high endogenous levels of Notch3 abolished VM both in vitro in Matrigel tube-formation assays (Figure 4c) and our 2D co-culture niche model (Figure $4 \mathrm{~d}$ ), and in vivo in tumorigenicity xenograft model (Figures 5d, f, and 6a,). Contrarily, Vartanian et al ${ }^{42}$ reported that blockage of Notch signaling with GSIs, DAPT, and dibenzazeoine, or Jag1 neutralizing Ab stabilizes VM in Mel Cher, Mel Kor, and Mel $\mathrm{P}$ melanoma cells in mono-culture and in mouse xenografts, indicating that Notch signaling attenuates VM. Moreover, Cui et $a l^{43}$ showed that Notch3 is downregulated during melanoma progression and ectopic expression of NICD3 in A375P melanoma cell line leads to growth inhibition through senescence in conventional 2D culture. Correspondingly, our data also demonstrated that Notch3 KD in A375 melanoma cells, exhibiting relatively low endogenous Notch 3 expression, failed to affect tumor growth (Figure 5a), MSLC frequencies (Figure 6c), and vascular/VM niche in vivo (data not shown). These discrepancies highlight the complexity and the importance of biological/cellular context of Notch signaling in melanoma. Indeed, a plethora of studies have concluded that the biological responses to Notch not only are cell 
type-, disease stage-, and context-specific, but also depend on the different receptor/ligand pairing. Divergent activities, both oncogenic and tumor suppressor function, have been described. ${ }^{23,44,45}$ Aside from the contextual diversity of Notch signaling, other possible explanations for the lack of response to Notch3 KD in A375 melanoma cells are the potential functional redundancy of different Notch receptors, and the possible involvement of signaling pathways other than Notch. In particular, a potential role for Notch4 warrants further investigation as concurrent Notch4 upregulation was observed in co-cultured melanoma cells in our in vitro niche model (Figure 2a) and Notch4 has been shown previously to control melanoma VM. ${ }^{24}$

Notch3 KD abolishes the MSLC niche morphogenesis not only by blocking VM phenotypic switch in 1205Lu melanoma cells, but also by inhibiting tumor angiogenesis as measured by the number and size of $\mathrm{CD}^{+} 1^{+}$murine vascular networks (Figure $5 \mathrm{~d}$ and $\mathrm{e}$ ), indicating Notch juxtacrine signaling between melanoma cells and stromal endothelium may mediate tumor angiogenesis. In concordance, Zeng et $a l^{46}$ showed that elevated Jag1 in head and neck squamous cell carcinoma triggers Notch activation in neighboring ECs and thereby promotes tumor angiogenesis. Of particular interest, similar Notch3-mediated tumor-stromal crosstalk is recently illuminated in the regulation of tumor dormancy and bone metastasis. Specifically, EC Dll4, induced by angiogenic factors in the tumor mircoenvironment, triggers Notch 3 activation in neighboring tumor cells, which then promotes a tumorigenic phenotype permitting escape from dormancy. ${ }^{47,48}$ In human breast cancer, interaction with osteoblasts in the bone marrow environment enhances Notch3 expression in the tumor cells, which accelerates bone metastasis. ${ }^{49}$ In accordance with these, upregulation of Dll1 in ECs in our in vitro niche model (Figure 2a) suggests that similar reciprocal melanoma-EC signaling may have a role.

In summary, we have identified Notch3 as a molecular switch controlling MSLC homeostasis and niche morphogenesis at least in a subset of melanomas. This is especially critical as effective targeting of MSLCs is compounded by their heterogeneous nature and genetic instability. As Notch3 signaling promotes vascular niche, which in turn drives 'dynamic stemness', Notch3-targeted approaches not only address the diverse pre-existing MSLC subsets by abolishing their vascular niche, but also prevent the induction of new MSLCs by blocking 'dynamic stemness'. With the availability of existing Notch signaling inhibitors currently available in the clinic for Alzheimer's disease and many others under development in the pharmaceutical industry, Notch signaling represents a novel and compelling target in melanoma. Of note, a phase 2 clinical trial using a GSI, RO4929097, showed minimal effects against metastatic melanoma likely due to inadequate drug levels as no significant inhibition of interleukin-2 (IL-2), a surrogate pharmacodynamic marker of Notch inhibition, was achieved in the peripheral blood T-cell assays. ${ }^{50}$ Nevertheless, considering the heterogeneity of melanoma both at clinico-pathologic and molecular/genetic levels, the expectation that a single agent will deliver significant treatment outcome in patients across the board is unrealistic. Nair et $a l^{51}$ recently showed that melanoma sensitivity to GSIs is dependent on functional phosphatase and tension homolog (PTEN). Furthermore, it is now appreciated that clinical response to GSIs also relies on endogenous Notch activities of the tumors. ${ }^{52,53}$ These studies, like ours, underline the contextual complexity of Notch signaling and may inform prospective genotyping or screening criteria for future patient selection. Combinatorial use of Notch-targeted strategies with cytotoxic agents has shown encouraging clinical efficacies in ovarian cancers ${ }^{53-56}$ and nasopharyngeal carcinoma. ${ }^{57}$ Hence, Notch-targeted approaches in combination with conventional or current state-of-the-art pathway-specific targeting strategies poise tremendous potentials as new therapeutic regimens in treating melanoma.

Supplementary Information accompanies the paper on the Laboratory Investigation website (http://www.laboratoryinvestigation.org)

\section{ACKNOWLEDGMENTS}

Dr Meenhard Herlyn (Wistar Institute, Philadelphia, PA, USA) is acknowledged for generously providing melanoma cell lines and reagents. This work was supported by the National Institutes of Health, National Cancer Institute grant no. R01CA138649, and a Research Scholars Award from the Department of Dermatology, Boston University School of Medicine, awarded to $\mathrm{M}-\mathrm{YH}$.

\section{DISCLOSURE/CONFLICT OF INTEREST}

The authors declare no conflict of interest.

1. Abel EV, Aplin AE. Finding the root of the problem: the quest to identify melanoma stem cells. Front Biosci (Schol Ed) 2011;3:937-945.

2. Shekhani MT, Jayanthy AS, Maddodi N, et al. Cancer stem cells and tumor transdifferentiation: implications for novel therapeutic strategies. Am J Stem Cells 2013;2:52-61.

3. Schatton T, Frank MH. Cancer stem cells and human malignant melanoma. Pigment Cell Melanoma Res 2008;21:39-55.

4. Fang D, Nguyen TK, Leishear $\mathrm{K}$, et al. A tumorigenic subpopulation with stem cell properties in melanomas. Cancer Res 2005;65: 9328-9337.

5. Somasundaram R, Villanueva J, Herlyn M. Intratumoral heterogeneity as a therapy resistance mechanism: role of melanoma subpopulations. Adv Pharmacol 2012;65:335-359.

6. Wicha MS. Cancer stem cells and metastasis: lethal seeds. Clin Cancer Res 2006;12:5606-5607.

7. Lai CY, Schwartz BE, Hsu MY. CD133+ melanoma subpopulations contribute to perivascular niche morphogenesis and tumorigenicity through vasculogenic mimicry. Cancer Res 2012;72:5111-5118.

8. Roesch A, Fukunaga-Kalabis M, Schmidt EC, et al. A temporarily distinct subpopulation of slow-cycling melanoma cells is required for continuous tumor growth. Cell 2010;141:583-594.

9. Shmelkov SV, Butler JM, Hooper AT, et al. CD133 expression is not restricted to stem cells, and both CD133+ and CD133- metastatic colon cancer cells initiate tumors. J Clin Invest 2008;118:2111-2120.

10. Park DM, Rich JN. Biology of glioma cancer stem cells. Mol Cells 2009;28:7-12.

11. Li Y, Laterra J. Cancer stem cells: distinct entities or dynamically regulated phenotypes? Cancer Res 2012;72:576-580.

12. Kumar SM, Liu S, Lu H, et al. Acquired cancer stem cell phenotypes through Oct4-mediated dedifferentiation. Oncogene 2012;31: 4898-4911. 
13. Li $Y$, Li A, Glas $M$, et al. c-Met signaling induces a reprogramming network and supports the glioblastoma stem-like phenotype. Proc Natl Acad Sci USA 2011;108:9951-9956.

14. Barcellos-Hoff MH, Newcomb EW, Zagzag D, et al. Therapeutic targets in malignant glioblastoma microenvironment. Semin Radiat Oncol 2009;19:163-170.

15. Denysenko T, Gennero L, Roos MA, et al. Glioblastoma cancer stem cells: heterogeneity, microenvironment and related therapeutic strategies. Cell Biochem Funct 2010;28:343-351.

16. Reardon DA, Wen PY. Therapeutic advances in the treatment of glioblastoma: rationale and potential role of targeted agents. Oncologist 2006;11:152-164.

17. Clarke MF, Becker MW. Stem cells: the real culprits in cancer? Sci Am 2006;295:52-59.

18. Herlyn M, Villanueva J. Sorting through the many opportunities for melanoma therapy. Pigment Cell Melanoma Res 2011;24:975-977.

19. Paulis YW, Soetekouw PM, Verheul HM, et al. Signalling pathways in vasculogenic mimicry. Biochim Biophys Acta 2010;1806:18-28.

20. Hendrix MJ, Seftor EA, Hess $A R$, et al. Vasculogenic mimicry and tumour-cell plasticity: lessons from melanoma. Nat Rev Cancer 2003;3: 411-421.

21. Folberg R, Hendrix MJ, Maniotis AJ. Vasculogenic mimicry and tumor angiogenesis. Am J Pathol 2000;156:361-381.

22. van der Schaft DW, Seftor RE, Seftor EA, et al. Effects of angiogenesis inhibitors on vascular network formation by human endothelial and melanoma cells. J Natl Cancer Inst 2004;96:1473-1477.

23. Liu J, Fukunaga-Kalabis M, Li L, et al. Developmental pathways activated in melanocytes and melanoma. Arch Biochem Biophys 2014;563:13-21.

24. Hardy KM, Kirschmann DA, Seftor EA, et al. Regulation of the embryonic morphogen Nodal by Notch4 facilitates manifestation of the aggressive melanoma phenotype. Cancer Res 2010;70: 10340-10350.

25. Qin JZ, Stennett L, Bacon P, et al. p53-independent NOXA induction overcomes apoptotic resistance of malignant melanomas. Mol Cancer Ther 2004;3:895-902.

26. Nickoloff BJ, Hendrix MJ, Pollock PM, et al. Notch and NOXA-related pathways in melanoma cells. J Investig Dermatol Symp Proc 2005;10: 95-104.

27. Howard JD, Moriarty WF, Park J, et al. Notch signaling mediates melanoma-endothelial cell communication and melanoma cell migration. Pigment Cell Melanoma Res 2013;26:697-707.

28. Hsu MY, Elder DE, Herlyn M. The Wistar melanoma (WM) cell lines. In: Masters J, Palsson B (eds). Hum Cell Culture, Vol 1. Kluwer: Nowell, MA USA, 1999, pp 259-274.

29. Schnegg $\mathrm{Cl}$, Yang $\mathrm{MH}$, Ghosh SK, et al. Induction of vasculogenic mimicry overrides VEGF-A silencing and enriches stem-like cancer cells in melanoma. Cancer Res 2015;75:1682-1690.

30. Mak AB, Schnegg C, Lai CY, et al. CD133-targeted niche-dependent therapy in cancer: a multipronged approach. Am J Pathol 2014;184: 1256-1262.

31. Andersson ER, Sandberg $R$, Lendahl U. Notch signaling: simplicity in design, versatility in function. Development 2011;138:3593-3612.

32. Huynh C, Poliseno L, Segura MF, et al. The novel gamma secretase inhibitor RO4929097 reduces the tumor initiating potential of melanoma. PLoS One 2011;6:e25264.

33. Arasada RR, Amann JM, Rahman MA, et al. EGFR blockade enriches for lung cancer stem-like cells through Notch3-dependent signaling. Cancer Res 2014;74:5572-5584.

34. Pasto A, Serafin V, Pilotto G, et al. NOTCH3 signaling regulates MUSASHI-1 expression in metastatic colorectal cancer cells. Cancer Res 2014;74:2106-2118.

35. Zhang Q, Lu C, Fang T, et al. Notch3 functions as a regulator of cell selfrenewal by interacting with the beta-catenin pathway in hepatocellular carcinoma. Oncotarget 2015;6:3669-3679.
36. Sansone $P$, Ceccarelli C, Berishaj M, et al. Self-renewal of CD133(hi) cells by IL6/Notch3 signalling regulates endocrine resistance in metastatic breast cancer. Nat Commun 2016;7:10442.

37. Yen WC, Fischer MM, Axelrod F, et al. Targeting Notch signaling with a Notch2/Notch3 antagonist (tarextumab) inhibits tumor growth and decreases tumor-initiating cell frequency. Clin Cancer Res 2015;21: 2084-2095.

38. Nickoloff BJ, Osborne BA, Miele L. Notch signaling as a therapeutic target in cancer: a new approach to the development of cell fate modifying agents. Oncogene 2003;22:6598-6608.

39. Liu ZJ, Xiao M, Balint K, et al. Notch1 signaling promotes primary melanoma progression by activating mitogen-activated protein kinase/phosphatidylinositol 3-kinase-Akt pathways and up-regulating $\mathrm{N}$-cadherin expression. Cancer Res 2006;66:4182-4190.

40. Balint K, Xiao M, Pinnix CC, et al. Activation of Notch1 signaling is required for beta-catenin-mediated human primary melanoma progression. J Clin Invest 2005;115:3166-3176.

41. Stine MJ, Wang CJ, Moriarty WF, et al. Integration of genotypic and phenotypic screening reveals molecular mediators of melanomastromal interaction. Cancer Res 2011;71:2433-2444.

42. Vartanian A, Gatsina G, Grigorieva I, et al. The involvement of Notch signaling in melanoma vasculogenic mimicry. Clin Exp Med 2013;13: 201-209.

43. Cui H, Kong $Y, X u M$, et al. Notch3 functions as a tumor suppressor by controlling cellular senescence. Cancer Res 2013;73:3451-3459.

44. Lobry C, Oh P, Mansour MR, et al. Notch signaling: switching an oncogene to a tumor suppressor. Blood 2014;123:2451-2459.

45. Danza G, Di Serio C, Rosati F, et al. Notch signaling modulates hypoxiainduced neuroendocrine differentiation of human prostate cancer cells. Mol Cancer Res 2012;10:230-238.

46. Zeng Q, Li S, Chepeha DB, et al. Crosstalk between tumor and endothelial cells promotes tumor angiogenesis by MAPK activation of Notch signaling. Cancer Cell 2005;8:13-23.

47. Indraccolo $S$. Insights into the regulation of tumor dormancy by angiogenesis in experimental tumors. Adv Exp Med Biol 2013;734: 37-52.

48. Indraccolo $\mathrm{S}$, Minuzzo $\mathrm{S}$, Masiero $\mathrm{M}$, et al. Cross-talk between tumor and endothelial cells involving the Notch3-DII4 interaction marks escape from tumor dormancy. Cancer Res 2009;69:1314-1323.

49. Zhang $\mathrm{Z}$, Wang $\mathrm{H}$, Ikeda $\mathrm{S}$, et al. Notch 3 in human breast cancer cell lines regulates osteoblast-cancer cell interactions and osteolytic bone metastasis. Am J Pathol 2010;177:1459-1469.

50. Lee SM, Moon J, Redman BG, et al. Phase 2 study of RO4929097, a gamma-secretase inhibitor, in metastatic melanoma: SWOG 0933. Cancer 2015;121:432-440.

51. Nair JS, Sheikh T, Ho AL, et al. PTEN regulates sensitivity of melanoma cells to RO4929097, the gamma-secretase inhibitor. Anticancer Res 2013;33:1307-1316.

52. Saito N, Fu J, Zheng S, et al. A high Notch pathway activation predicts response to gamma secretase inhibitors in proneural subtype of glioma tumor-initiating cells. Stem Cells 2014;32:301-312.

53. Morgan SL, Wyant GA, Dinulescu DM. 'Take it up a NOTCH': novel strategies for cancer therapy. Cell Cycle 2013;12:191-192.

54. Kang $\mathrm{H}$, Jeong JY, Song JY. et al. Notch3-specific inhibition using siRNA knockdown or GSI sensitizes paclitaxel-resistant ovarian cancer cells. Mol Carcinog 2016;55:1196-1209.

55. Park JT, Chen X, Trope CG, et al. Notch3 overexpression is related to the recurrence of ovarian cancer and confers resistance to carboplatin. Am J Pathol 2010;177:1087-1094.

56. McAuliffe SM, Morgan SL, Wyant GA, et al. Targeting Notch, a key pathway for ovarian cancer stem cells, sensitizes tumors to platinum therapy. Proc Natl Acad Sci USA 2012;109:E2939-E2948.

57. Man $\mathrm{CH}$, Wei-Man Lun S, Wai-Ying Hui J, et al. Inhibition of NOTCH3 signalling significantly enhances sensitivity to cisplatin in EBV-associated nasopharyngeal carcinoma. J Pathol 2012;226:471-481. 